\title{
La Gestión de la colección en las bibliotecas universitarias españolas. Planes y normativas (II): la adquisición
}

\author{
Jose Luis Herrera-Morillas* \\ *Universidad de Extremadura. Departamento de Información y Comunicación. \\ Correo-e: jlhermor@unex.es
}

Recibido: 11-12-2012; 2a version: 14-02-2013; Aceptado: 15-03-2013.

\begin{abstract}
Cómo citar este artículo/Citation: Herrera-Morillas, J. L. (2014). La Gestión de la colección en las bibliotecas universitarias españolas. Planes y normativas (II): la adquisición. Revista Española de Documentación Científica, 37(1):e030. doi: http:// dx.doi.org/10.3989/redc.2014.1.1051

Resumen: Este artículo recoge la segunda parte del trabajo de investigación dedicado al estudio de la gestión de la colección en las bibliotecas universitarias españolas a partir del análisis de los planes y normativas elaborados por estas bibliotecas. La primera parte se tituló: La gestión de la colección en las bibliotecas universitarias españolas. Planes y normativas (I): la selección. Ahora se estudian treinta textos que regulan la adquisición de recursos de información en las bibliotecas universitarias españolas. Se describe la adquisición como parte de la gestión de la colección según se recoge en los documentos; prestando especial atención a la estructura y contenidos de los documentos, a su concepto y objetivos. En segundo lugar, se profundiza en cómo se plantea el procedimiento de la adquisición: aparece como un proceso diferenciado y a la vez relacionado con el de la selección, estructurado y normalizado, que presta atención a las donaciones y notablemente vinculado a las publicaciones impresas, pero en el contexto de una biblioteca híbrida.
\end{abstract}

Palabras clave: Gestión de la colección; adquisición; bibliotecas universitarias; España; normativas; planes.

\section{Collection management in Spanish university libraries. Plans and regulations (II): acquisitions}

Abstract: This article presents the second part of an investigative work dedicated to study collection management in Spanish university libraries through an analysis of the plans and regulations elaborated by these libraries. The first part was entitled: Collection management in Spanish university libraries. Plans and regulations (I): selection. In the present work thirty texts are studied which regulate the acquisition of information resources at Spanish university libraries. These documents describe acquisitions as part of collection management and special attention is focused on their structure and contents, as well as on their concept and objectives. Secondly the study delves into the acquisitions procedure. It appears differentiated from selection, but at the same time, related to it. It is structured and standardized, and includes donations. Although principally linked to print publications, the results nonetheless are presented within the context of a hybrid library.

Keywords: Collection management; acquisitions; university libraries; Spain; regulations; plans.

Copyright: () 2014 CSIC. Este es un artículo de acceso abierto distribuido bajo los términos de la licencia Creative Commons Attribution-Non Commercial (by-nc) Spain 3.0. 


\section{INTRODUCCIÓN}

Este artículo recoge la segunda parte del trabajo de investigación dedicado al estudio de la gestión de la colección en las bibliotecas universitarias españolas a partir del análisis de los planes y normativas elaborados por estas bibliotecas (HerreraMorillas 2013).

La primera parte se dedicó a dar una visión de conjunto de esta política de gestión según se desprende de los documentos localizados y a analizar con más detalle uno de sus aspectos: la selección. De las setenta y una bibliotecas universitarias localizadas se detectaron treinta y tres bibliotecas con documentos relacionados con la gestión de la colección que constituyeron la fuente de estudio.

Ahora, como continuación de esta primera parte, se aborda el estudio de la adquisición.

"Adquirir consiste en ingresar en la biblioteca los materiales seleccionados, en el menor tiempo posible y en las mejores condiciones, para ponerlos a disposición de los usuarios" (Moreno, 2002).

Para Fuentes (2010) el proceso de adquisición es una consecuencia directa del proceso de selección y sólo desde un buen proceso de selección se podrá llegar a alcanzar la eficacia en las actuaciones de la adquisición, que abarcan:

"Toda una serie de fases tales como la petición de materiales, la facturación, la recepción, la comprobación, el pago y cualquier otra operación relacionada con el proceso total".

La literatura sobre la adquisición localizada versa, en gran medida, sobre el acceso y otros usos relacionados con la gestión de la colección electrónica: cuando los formatos electrónicos empiezan a tener presencia en las bibliotecas aparece la preocupación por el coste de las colecciones electrónicas (Cassell, 2004) y se realizan estudios para ver las prácticas en las bibliotecas sobre las licencias y políticas de acceso (Horava, 2005); ya en nuestros días la tendencia más novedosa se centra en el papel que las publicaciones en acceso abierto pueden tener en el desarrollo de la colección (Yundi, 2006; Rodríguez, 2007; Borgoños, 2010).

Con anterioridad, la gestión y la mejora de los procesos relacionados con la adquisición de colecciones bibliográficas también ha sido objeto de atención. Es el caso de la completa publicación de Osburn (1992), que dentro de un contexto amplio aborda el presupuesto de adquisiciones y su relación con la evaluación de colección; de los trabajos de Calenge (1994, 1999) que, tras una serie de experiencias y encuestas, apoya la necesidad de una política de gestión formalizada y la conveniencia de identificar etapas funcionales en la política de adquisiciones; también subraya que se debe distinguir el momento de la selección de las decisiones sobre la adquisición y analiza las implicaciones de esta concepción dentro de los procesos de constitución de la colección; o del texto de Wilkinson y Thorson (1998) dedicado a la aplicación del proceso RFP como estrategia eficaz para la gestión de las adquisiciones. Mención aparte supone la nueva situación que se inicia cuando se empiezan a imponer los sistemas automatizados (Boss, 1980, 1982).

La adquisición de materiales impresos se ha analizado en trabajos de carácter más localista, como el de Paulos y Holley (2006), que aborda la relación entre los programas de estudios africanos y la adquisición de impresos africanos en cuatro bibliotecas académicas americanas o el de Dali y Dilevko (2005), centrado en la adquisición de libros en países eslavos de la Europa del Este, en relación con bibliotecas norteamericanas.

\section{MATERIAL Y MÉTODO}

La metodología utilizada en este trabajo se asemeja mucho a la empleada en la primera parte. En este caso, se analizan los contenidos incluidos en los textos recopilados desde el punto de vista de la adquisición. Previamente, se ha realizado, de nuevo, una visita a los sitios web en el periodo comprendido entre el 15 de noviembre y el 10 de diciembre de 2012 con el objeto de verificar la actualidad de la información.

Los datos extraídos del análisis/estudio se han estructurado en siete apartados:

$$
\begin{aligned}
& \text { - Estructura y contenidos de los textos. } \\
& \text { - Concepto de adquisición. } \\
& \text { - Formas o modalidades de adquisición. } \\
& \text { - Criterios para la adquisición. } \\
& \text { - El presupuesto. } \\
& \text { - Las donaciones. } \\
& \text { - Los proveedores. }
\end{aligned}
$$

\section{RESULTADOS Y DISCUSIÓN}

En este apartado se presentan los resultados y discusión de los datos obtenidos del análisis de diez planes de gestión de la colección, once normativas y documentos específicos sobre la adquisición y quince sobre las donaciones. Para ello se siguen los siete apartados expuestos en el punto anterior.

Los apartados uno al cinco y el sexto hacen referencia a los planes de gestión de la colección y a las normativas o documentos específicos sobre la adquisición. El apartado seis, además, incluye los datos extraídos de los documentos específicos sobre donaciones.

\subsection{La adquisición en los planes y normati- vas: estructura y contenido de los textos}

De los diez planes de gestión de la colección, en cuatro (Carlos III, La Rioja, Málaga y Sevilla) se 
aborda la adquisición junto a la selección dentro de una misma política, aunque diferenciando ambos procesos. Todos los planes coinciden en incluir como contenido destacado dentro de la adquisición, las formas o modalidades de adquisición. Esta información se completa con otras como:

- Política de proveedores (La Rioja y Murcia).

- Tipos de documentos (Sevilla y Murcia).

- Criterios específicos para la compra de monografías (Barcelona).

- Acuerdos de cooperación (Murcia).

- Áreas prioritarias de adquisición (Sevilla).

De las once bibliotecas que abordan la adquisición en documentos específicos, cinco bibliotecas (Alicante, Burgos, Ávila, Huelva y Politécnica de Cartagena) presentan los documentos redactados en forma de articulado. En dos bibliotecas (Extremadura y La Laguna) la adquisición se incluye en manuales de procedimiento o de procesos. La Biblioteca de Cantabria presenta la información sobre la adquisición en un apartado de la página web (descripción del servicio, procedimientos de compra, etc.). El resto de los textos están compuestos por diferentes capítulos o apartados (Castilla-La Mancha, Pablo Olavide y Pontificia de Comillas).

La Universidad de Alicante y la Politécnica de Cartagena, además, han elaborado normativas para la suscripción de bases de datos y publicaciones periódicas.

En los once documentos mencionados los contenidos se explicitan de forma escueta con textos de extensión reducida que se limitan a indicar:

- Distribución del presupuesto (Alicante).

- Tramitación y presentación de peticiones (Huelva).

- Instrucciones prácticas sobre la distribución del presupuesto y las propuestas de adquisiciones (Pablo Olavide y Politécnica de Cartagena).

- Descripción de la constitución de la colección y criterios generales para la adquisición bibliográfica (Burgos y Católica de Ávila).

- Descripción del "servicio" de adquisiciones (Cantabria).

Y también, de forma más desarrollada, como en los documentos de Castilla La Mancha -que además de los temas anteriores incluye la política de donaciones e intercambio, los servicios de las secciones de adquisiciones y las bajas en inventario-; - los de Extremadura y La Laguna, en ambos casos, centrados en la descripción técnica y práctica de los procedimientos.

\subsection{Concepto de adquisición}

El concepto de adquisición aparece recogido en dos planes generales de gestión de la colección
(Barcelona y Murcia), y en cuatro documentos sobre la adquisición (Burgos, Extremadura, Huelva y Politécnica de Cartagena). En estas definiciones observamos cómo se incide en el carácter de "tramitación" y "gestión" por medio de "tareas" y "rutinas" con el fin de "incorporar" el material seleccionado:

"Consiste en la tramitación de las obras seleccionadas mediante tareas y rutinas para incorporarlas a la colección por el procedimiento más adecuado. Su objeto es conseguir los materiales en la cantidad y calidad deseada al menor coste y tiempo, es decir lograr la eficiencia" (Universidad de Murcia)

La Universidad de Barcelona se aparta un poco de ese planteamiento al concebir la adquisición como una medida para cubrir las necesidades bibliográficas de la docencia y la investigación, en función de tres categorías de documentos.

La Biblioteca de la Universidad de Málaga hace la siguiente matización:

"Actualmente, cabría hablar de 'incorporación' de documentos a la colección, pues la aparición de documentos digitales ha alterado el procedimiento clásico de adquisición de documentos".

También, es frecuente que la adquisición no se defina y aparezca planteada directamente en las formas o modalidades de adquisición (Autónoma de Barcelona, Carlos III, La Rioja, Politécnica de Cataluña, Sevilla y Castilla La Mancha).

No cabe duda que la adquisición, como afirma Moreno (2002), debe ser considerada "un proceso bibliotecario fundamental", consume gran parte del presupuesto de la biblioteca y "por lo tanto debe ser gestionado por bibliotecarios". Por eso es muy conveniente, tal como establece REBIUN (1999), que las bibliotecas tengan una sección de adquisiciones para "coordinar y supervisar la coherencia y equilibrio de las colecciones que se adquieran".

\subsection{Formas o modalidades de adquisición}

Las formas o modalidades de adquisición se mencionan en nueve planes generales de gestión de la colección (tabla I). Todos coinciden en incluir las modalidades de donación, intercambio (o canje) y compra (la Complutense no la menciona expresamente pero se sobrentiende).

En la compra, algunos documentos diferencian varias modalidades, y hay textos que citan otras modalidades de adquisición junto a las anteriores.

La tendencia más novedosa es, sin duda, la de incluir los recursos en Internet de acceso gratuito como los repositorios institucionales para incrementar la colección. Se debe contar con ellos debido a que se utilizan cada vez más para difundir información científica en Internet e incluir en ellos, normalmente con acceso libre, "los documentos digitales o digitalizados que forman los fondos y 
Tabla I. Formas o modalidades de adquisición

\begin{tabular}{|c|c|}
\hline Universidad (Biblioteca) & Formas o modalidades de adquisición \\
\hline Autónoma de Barcelona & $\begin{array}{l}\text {-Compra } \\
\text {-Donación } \\
\text {-Intercambio } \\
\text {-Depósito } \\
\text {-Licencia } \\
\text {-Acceso libre en Internet }\end{array}$ \\
\hline Barcelona & $\begin{array}{l}\text {-Compra } \\
\text {-Donaciones } \\
\text {-Intercambio de publicaciones } \\
\text {-Depósito Legal }\end{array}$ \\
\hline Carlos III & $\begin{array}{l}\text {-Compra } \\
\text {-Donación } \\
\text {-Canje } \\
\text {-Depósitos }\end{array}$ \\
\hline Complutense & $\begin{array}{l}\text { (Compra, se sobreentiende) } \\
\text {-Donación } \\
\text {-Canje } \\
\text {-Suministro de documentos y préstamo interbibliotecario } \\
\text { Recursos electrónicos } \\
\text {-Adquisición coordinada } \\
\text {-Adquisición cooperativa }\end{array}$ \\
\hline La Rioja & $\begin{array}{l}\text {-Compra } \\
\text {-Donación } \\
\text {-Intercambio o canje de publicaciones } \\
\text {-Depósito } \\
\text {-Suscripción }\end{array}$ \\
\hline Málaga & $\begin{array}{l}\text { A) Adquisición de material físico } \\
\text {-Compra } \\
\text {-Donaciones } \\
\text {-Intercambio entre universidades } \\
\text {-Canje } \\
\text {-Depósito } \\
\text { B) Acceso a los documentos (formato electrónico) } \\
\text {-Contratación de recursos electrónicos mediante suscripción o compra, directamente } \\
\text { por parte de la BUMA o a través de los Consorcios en que participe } \\
\text {-Recursos en Internet de acceso gratuito } \\
\text { C) Incorporación de la producción de documentos docentes y de investigación } \\
\text { propios a través de repositorios institucionales }\end{array}$ \\
\hline Murcia & $\begin{array}{l}\text {-Compra } \\
\text {-Donativos } \\
\text {-Canje } \\
\text {-Compartida o cooperativa } \\
\text { Revistas y recursos electrónicos } \\
\text {-Concurso público anual } \\
\text {-Procedimiento negociado } \\
\text {-Compra consorciada }\end{array}$ \\
\hline Politécnica de Cataluña & $\begin{array}{l}\text {-Compra } \\
\text {-Donativo } \\
\text {-Intercambio }\end{array}$ \\
\hline Sevilla & $\begin{array}{l}\text {-Compra directa } \\
\text {-Donativo } \\
\text {-Canje } \\
\text {-Concurso de suministro } \\
\text {-Consorcio del CBUA }\end{array}$ \\
\hline
\end{tabular}


colecciones relacionados con una unidad de información" (Llueca, 2011). Sin embargo, sólo los mencionan dos universidades (Autónoma de Barcelona y Málaga) y esto contrasta con el hecho de que gran parte de ellas han creado y mantienen repositorios institucionales donde albergan los documentos fruto de actividades docentes e investigadoras de sus propias instituciones (Anglada y otros, 2005).

La mención expresa a los recursos electrónicos se hace en tres universidades (Complutense, Málaga y Murcia) - sin tener en cuenta la normativas para la suscripción de bases de datos y publicaciones periódicas de la Universidad de Alicante y la Politécnica de Cartagena-, y esto choca con el hecho de que hoy es difícil encontrar una biblioteca que tenga sólo materiales impresos (Yeow-Fei, 2004).

Estas consideraciones inducen a afirmar que la Universidad de Málaga es la que presenta un listado más claro, completo y actualizado de formas o modalidades de adquisición.

\subsection{Criterios para la adquisición}

Los planes generales de gestión de la colección de las universidades de Barcelona, Carlos III y Complutense, son los únicos que incluyen criterios a tener en cuenta en la adquisición.

La Universidad de Barcelona presenta unos criterios cuantitativos:

- Bibliografía recomendada básica de obras y manuales para el alumnado de primer y segundo ciclo:

- Menos de 100 alumnos: 2 ejemplares.

- De 100 a 299 alumnos: de 3 a 4 ejemplares.

- De 300 a 499 alumnos: de 4 a 6 ejemplares.

- De 500 a 599 alumnos: de 6 a 10 ejemplares.

- Más de 600 alumnos: de 7 a 12 ejemplares.

- Bibliografía recomendada complementaria

- 2 ejemplares, máximo (si la obra es en catalán, en castellano o en la lengua de las asignaturas, caso de Filología).

- 1 ejemplar en el caso de otros idiomas.

- Obras para tercer ciclo, profesorado y personal investigador

- 1 ejemplar de los libros solicitados por el personal docente o investigador de la misma universidad.

- Obras de referencia

- Generales: se intentará tener la última edición.
- Específicas: se dará preferencia a la actualización de este tipo de obras de referencia respecto a las generales.

- Siempre que se pueda se comprará la versión electrónica en red.

La Universidad Carlos III ha fijado este baremo para los manuales:

- Menos de 100 alumnos: 2 ejemplares.

- Más de 100 alumnos: 3 ó 4 ejemplares.

- Más de 300 alumnos: 4 a 6 ejemplares.

- Más de 500 alumnos: 5 a 8 ejemplares.

- Más de 600 alumnos: 6 a 10 ejemplares.

- Más de 700 alumnos: 7 a 12 ejemplares.

. 800 alumnos: 12 ejemplares.

La Universidad Complutense de Madrid señala que la Biblioteca debe adquirir materiales documentales de todas y cada una de las disciplinas de las que se imparten enseñanzas, teniendo en cuenta que estén presentes esta variedad de documentos:

- Bibliografía básica.

- Colecciones de investigación.

- Obras de referencia.

- Otras colecciones (según la disponibilidad económica).

También establece condiciones sobre:

- Lengua de los documentos.

- Tipología y formatos.

- Duplicación.

- Criterios típicos.

Los criterios cuantitativos son de utilidad para guiar la adquisición, sobre todo, cuando se está empezando de cero la formación de una colección o hay que incrementarla por la incorporación de nuevas titulaciones. Se deben complementar con criterios cualitativos en los que deben jugar un papel fundamental los docentes e investigadores que trabajan en la universidad donde se ubica la biblioteca, por ejemplo, que los responsables de las adquisiciones conozcan con antelación suficiente las bibliografías que van a recomendar en sus asignaturas.

\subsection{El presupuesto}

El presupuesto se aborda en trece textos (seis planes generales de gestión de la colección y siete documentos específicos sobre la adquisición). Los contenidos se centran en el reparto del presupuesto (siete documentos) y en las partidas presupuestarias o procedencia de los recursos (cinco documentos). 
El tema del presupuesto se destaca como apartado principal del índice en cinco planes generales de gestión de la colección, normalmente bajo la denominación de "Política presupuestaria" (Carlos III, Murcia, La Rioja y Politécnica de Cataluña).

Los documentos que le dedican una mayor extensión son el de la Universidad de La Rioja (órganos responsables de la política de adquisiciones y presupuestaria; partidas presupuestarias; plazos de aprobación del presupuesto y de reparto del mismo; bases de ejecución del presupuesto; políticas de remanentes; recomendaciones a los departamentos propuestas para la ejecución del presupuesto de bibliografía; criterios de reparto del presupuesto de adquisición de fondos; procedimientos de gestión de presupuestos de las adquisiciones bibliográficas); el de la Universidad Complutense (financiación de las colecciones en relación al presupuesto general de la Universidad; las diferentes partidas presupuestarias que intervienen); y el de la Universidad de Castilla La Mancha (nueve normas para la "asignación de presupuestos de adquisición centralizada de materiales bibliográficos").

Cuando son variadas las fuentes presupuestarias para adquirir fondos documentales en una misma universidad, es fundamental que exista una eficaz coordinación para evitar las duplicaciones y solapamientos innecesarios en las adquisiciones, se deben arbitrar mecanismos para evitar ese peligro, por ejemplo, que todos los fondos, independientemente de su fuente de financiación, sean inventariados y catalogados por la biblioteca, y que cualquier adquisición duplicada sea convenientemente justificada.

\subsection{Las donaciones}

Las donaciones se incluyen en nueve planes de gestión de la colección del siguiente modo: cuatro textos recogen una definición de las donaciones (Carlos III, La Rioja, Complutense y Murcia). En ocho textos se matizan las donaciones con unas condiciones generales (Autónoma de Barcelona, Carlos III, Complutense, Sevilla y Murcia) o criterios y recomendaciones más detallados (Barcelona y Politécnica de Cataluña). En cuatro textos se establecen tipos de donaciones (Barcelona, Carlos III, Complutense y La Rioja). El texto de la Universidad Carlos III se detiene en exponer el procedimiento a seguir para las grandes donaciones, también el de La Rioja. El texto de Málaga solo las cita como uno más de los procedimientos de adquisición. El texto de la Universidad Alfonso X El Sabio no las menciona.

De los once textos dedicados a la adquisición, ocho hacen referencia a las donaciones. De ellos, tres (Burgos, Cantabria y La Laguna), solamente las mencionan. Los textos que les dedican una mayor atención son el de Castilla-La Mancha, con el capítulo "Política de donaciones e intercambio"; el de Extremadura, que incluye dentro del apartado general "Desarrollo del proceso" un subapartado para la "Incorporación de monografías por donación e intercambio" para abordar la gestión de donaciones (problemas de gestión que plantean, procedencias de las donaciones), y la gestión de duplicados procedentes de la donación; y el texto de la Universidad Pablo Olavide con varias orientaciones y criterios para que sirvan de ayuda en esta tarea. Los demás textos son el de la Universidad de Ávila, que las nombra como una forma de adquisición y les dedica un artículo donde se marcan algunas condiciones, al igual que sucede con la Universidad de Comillas.

Los documentos específicos dedicados a las donaciones son quince ${ }^{1}$. Suelen ser textos breves, sólo cinco documentos superan las tres páginas (Valladolid nueve; Lleida siete; Vigo siete; y Rey Juan Carlos seis). El contenido más común hace referencia al procedimiento o pasos a seguir (trece documentos); le siguen los criterios que se deben tener en cuenta (doce documentos) (tabla II); los tipos de donaciones (cinco documentos) (tabla III); y la definición de donación (tres documentos: Las Palmas de Gran Canaria, Rey Juan Carlos y Valladolid).

Es lógico este tratamiento diferenciado de las donaciones, pues hay que considerarlas como una forma alternativa de adquisición y por consiguiente "el procedimiento de selección podrá ser diferente" (Fuentes, 2010).

\subsubsection{Definición de donaciones}

El concepto de donaciones aparece definido en siete textos (cuatro planes generales de de gestión de la colección: Carlos III, Complutense, La Rioja y Murcia; y tres documentos específicos sobre donaciones: Las Palmas de Gran Canaria, Rey Juan Carlos y Valladolid).

Las definiciones coinciden en indicar la gratuidad del procedimiento aunque matizando que implica una serie de costes referidos a la gestión y proceso técnico:

"Es una forma de adquisición gratuita en la Biblioteca pero el proceso del material para su puesta a disposición del público implica una serie de costes en recursos humanos y problemas de almacenamiento, por lo tanto se hace necesario establecer unas condiciones mínimas para la aceptación de donaciones" (Universidad Carlos III).

Los documentos de la Universidad Complutense y de la Universidad de Valladolid recogen la misma definición:

"Es la cesión gratuita de un documento o conjunto de documentos y una forma de adquisición gratuita en las bibliotecas. Sin embargo, el proceso técnico de las obras donadas para su puesta a disposición del público implica una serie de costes (personal, presupuesto, espacios) y la integración de estas obras en las bibliotecas puede conducir a un crecimiento descontrolado de la colección, por lo que es necesario establecer unas condiciones para la aceptación de donaciones". 
Esta gratuidad hace que algunos autores tiendan a agrupar las donaciones y el canje en el mismo sector dentro de la estructura organizativa, debido a que no suponen importantes gastos (Aguado, 2011). En el caso de los textos analizados se diferencian claramente.

En estas definiciones el contenido de las donaciones suele ser una oferta de materiales que se hace a la biblioteca para incrementar los fondos, pero también se debería hacer mención a la posibilidad de recibir una oferta de dinero destinado al mismo fin (Aguado, 2011). Esta tendencia es interesante y se debería potenciar para contrarrestar los recortes presupuestarios. Las donaciones no se deben contemplar como un procedimiento "pasivo" donde la iniciativa siempre parte del donante, sino que debe evolucionar hacia una práctica en la que también sea frecuente que la iniciativa parta de la biblioteca, enmarcada en las prácticas del fundraising.

\subsubsection{Criterios}

Los criterios se incluyen en quince documentos. Si se analizan, se observa cierta variedad en cuanto a la redacción y contenido (tabla II). Los criterios en los que más coinciden son los siguientes:

- Obras en buen estado (doce documentos).

- Obras no duplicadas, salvo excepciones (ocho documentos).

- Obras adecuadas y de interés para la enseñanza que se imparte (siete documentos).

- Obras adecuadas y de interés para la investigación (seis documentos).

- Libros antiguos o de valor cultural y bibliográfico (cinco documentos).

- Obra propias de las materias o especialización de la biblioteca (tres documentos).

Los criterios que presentan los textos de Barcelona y Lleida son idénticos, al igual que los de Granada y Extremadura por un lado, y los de Navarra e Internacional de Cataluña por otro.

Los libros antiguos solo se mencionan en los textos de cinco universidades (Extremadura, Granada, Internacional de Cataluña, Jaén y Navarra) que los incluyen como uno de los criterios.

El interés por que se apliquen unos determinados criterios refleja la inquietud de que toda donación implica un costo para la biblioteca desde que se acepta hasta que se pone a disposición de los usuarios y por ello, no se pude aceptar cualquier cosa, y sobre todo, algo que nunca hubiera sido objeto de una compra por no estar relacionado con los objetivos de la biblioteca.

Sin embargo, se echa en falta un mayor detenimiento respecto a la evaluación de la disponibilidad de recursos para financiar los gastos técnicos y de personal. A este aspecto La IFLA en sus directrices
Donaciones para las Colecciones: Directrices para las Bibliotecas (2010) da bastante importancia a estos gastos, indicando que se deben considerar los siguientes costos:

- Adquisición (preparación para el traslado, etc.).

- Procesamiento.

- Organización del acceso.

- Preservación.

- Ubicación-almacenamiento.

\subsubsection{Procedimiento}

El procedimiento para llevar a cabo una donación se explicita en dieciséis documentos. Los pasos básicos más frecuentes son los que siguen:

- El donante se debe poner en contacto con la biblioteca (siete documentos).

- El donante debe aportar información sobre el contenido de la donación (once documentos).

- La toma de decisión: aceptación o rechazo (ocho documentos).

- La biblioteca debe elaborar un informe (sobre el interés de la donación y manifestarse favorable o desfavorable) (cinco documentos).

- La entrega por parte del donante de un catálogo u otros instrumentos de descripción bibliográfica que tenga disponibles, de los documentos a donar (dos documentos).

- Las donaciones aceptadas pasan a ser propiedad de la biblioteca (cinco documentos).

- El envío de una carta de agradecimiento al donante (cuatro documentos).

En algunos textos se precisa que previamente el donante debe comprobar en el catálogo de la biblioteca si tiene esos documentos (cinco documentos). Varios textos incluyen algunas indicaciones referentes a la posterioridad de la incorporación de las donaciones como propiedad de la biblioteca (cuatro documentos).

En relación con la toma de decisión sobre la aceptación o rechazo de la donación, la IFLA en sus directrices Donaciones... (2010), aporta el tipo de información que se debe reunir para facilitar el proceso de toma de decisiones. Incluye estos aspectos:

- Propiedad.

- Materiales con información delicada.

- Procedencia.

- Motivo de la donación.

- Adecuación de los materiales ofrecidos.

- Condiciones especiales.

La fijación de un procedimiento es necesaria para conseguir que la donación sea útil, legal e impida 
Tabla II. Criterios para donaciones

\begin{tabular}{|c|c|}
\hline \multicolumn{2}{|c|}{ Universidad (Biblioteca) $\quad$ Criterios para donaciones } \\
\hline \multicolumn{2}{|c|}{ PLANES DE GESTIÓN DE LA COLECCIÓN } \\
\hline Barcelona & $\begin{array}{l}\text {-Obras propias de las materias de la biblioteca con un nivel universitario } \\
\text {-Obras que se adecúen a las necesidades de cada enseñanza } \\
\text {-Obras en las que hayan participado profesores de la Universidad de la temática de la Biblioteca } \\
\text {-Obras de creación literaria que sean interesantes para la biblioteca y estén escritas con rigor descriptivo }\end{array}$ \\
\hline Complutense & $\begin{array}{l}\text {-Estado de conservación (en buen estado, salvo en material histórico) } \\
\text { - Existencia (no duplicados, salvo excepciones) } \\
\text {-Vigencia } \\
\text { - Materia (relación con programas de estudios y líneas de investigación) } \\
\text { - Copias (no reproducciones) } \\
\text {-Publicaciones periódicas (en relación con la colección) }\end{array}$ \\
\hline $\begin{array}{l}\text { Politécnica de } \\
\text { Cataluña }\end{array}$ & $\begin{array}{l}\text {-Duplicados (se admiten en casos excepcionales) } \\
\text { - Ámbito de especialización (adecuarse a la especialización de la biblioteca) } \\
\text { - Contenido (no se aceptan resúmenes o documentos abreviados y estrictos con las separatas y folletos) } \\
\text { - Continuidad (si forma parte de una serie que completa la colección) } \\
\text { - Originalidad (tienen que estar editados) } \\
\text {-Estado físico (adecuado) } \\
\text {-Idioma (puede ser un elemento a tener en cuenta) } \\
\text {-Obsolescencia }\end{array}$ \\
\hline \multicolumn{2}{|c|}{ DOCUMENTOS ESPECÍFICOS SOBRE DONACIONES } \\
\hline Extremadura & $\begin{array}{l}\text { Se valorarán especialmente: } \\
\text {-Libros raros, antiguos, curiosos, o que tengan una especial relación con la Comunidad Autónoma de Extremadura } \\
\text {-Bibliotecas de profesores universitarios, de bibliófilos y en general, bibliotecas de temática especializada aunque no sea muy } \\
\text { amplio el fondo que contengan } \\
\text { - Colecciones de revistas } \\
\text {-Archivos antiguos o de personajes relevantes de la historia reciente }\end{array}$ \\
\hline Granada & $\begin{array}{l}\text {-Libros antiguos, hasta el año } 1900 \text { inclusive } \\
\text {-Bibliotecas de profesores universitarios, de bibliófilos y, en general, bibliotecas de tema especializado, aunque no sea muy } \\
\text { amplio el fondo que contengan, siempre que interesen y exista espacio físico para ubicarlos. } \\
\text {-Colecciones de revistas, que sean de interés o permitan completar colecciones existentes }\end{array}$ \\
\hline $\begin{array}{l}\text { Internacional } \\
\text { de Cataluña }\end{array}$ & $\begin{array}{l}\text {-Libros antiguos (anteriores a 1820) } \\
\text { - Bibliotecas de tema especializado, aunque no sea muy amplio el fondo que contengan } \\
\text { - Colecciones de revistas } \\
\text {-Archivos antiguos o de personajes relevantes de la historia reciente } \\
\text { - Libros en buen estado (salvo antiguos o de especial valor) } \\
\text { - No interesan libros del área de ciencias experimentales anteriores a 1980, salvo que tengan especial valor por la dedicatoria, } \\
\text { el autor u otras circunstancias }\end{array}$ \\
\hline Jaén & $\begin{array}{l}\text {-Libros antiguos (anteriores a 1900) } \\
\text { - Bibliotecas de profesores universitarios, bibliófilos y en general, bibliotecas de tema especializado, aunque no sea muy amplio } \\
\text { el fondo que contengan } \\
\text {-Libros en buen estado }\end{array}$ \\
\hline $\begin{array}{l}\text { Las Palmas de } \\
\text { Gran Canaria }\end{array}$ & $\begin{array}{l}\text { - Conservación (material en buen estado, salvo en fondo antiguo) } \\
\text { - Existencia (no duplicados, salvo excepciones) } \\
\text {-Materia (temática de los planes de estudio o líneas de investigación) } \\
\text {-Publicaciones periódicas (se descartan números que no están en la colección) }\end{array}$ \\
\hline Lleida & $\begin{array}{l}\text {-Obras propias de las materias de la biblioteca con un nivel universitario } \\
\text {-Obras que se adecúen a las necesidades de cada en enseñanza } \\
\text {-Obras en las que hayan participado profesores de la Universidad de la temática de la Biblioteca } \\
\text {-Obras de creación literaria sean interesantes para la biblioteca y estén escritas con rigor descriptivo }\end{array}$ \\
\hline Navarra & $\begin{array}{l}\text { - Libros antiguos (anteriores a 1835) } \\
\text { - Bibliotecas de tema especializado, aunque no sea muy amplio el fondo que contengan } \\
\text { - Colecciones de revistas } \\
\text { - Archivos antiguos o de personajes relevantes de la historia reciente } \\
\text { - Libros en buen estado (salvo antiguos o de especial valor) } \\
\text { - No interesan libros del área de ciencias experimentales anteriores a 1980, salvo que tengan especial valor por la dedicatoria, } \\
\text { el autor u otras circunstancias }\end{array}$ \\
\hline Pompeu Fabra & $\begin{array}{l}\text { Obras en buen estado físico, que sean de interés para la docencia y la investigación que se realizan en la Universidad y que no } \\
\text { se incluyan dentro de los siguientes supuestos: } \\
\text {-Documentos duplicados, ya existentes en los fondos } \\
\text {-Documentos en mal estado } \\
\text { - Documentos con contenido no apropiado al nivel académico de una biblioteca universitaria } \\
\text {-Documentos con contenidos temáticos no apropiados a la UPF } \\
\text {-Documentos con contenidos obsoletos, actualizados por publicaciones posteriores } \\
\text {-Documentos en formatos obsoletos (VHS, beta, disquetes, discos de vinilo, etc.) } \\
\text { - Publicaciones seriadas no completas o que no completen la colección de la UPF } \\
\text { - Obras en volúmenes que no están completas o que no completan la colección de la UPF } \\
\text {-Documentos que se pueden encontrar en formato electrónico de acceso no restringido } \\
\text {-Tesis o trabajos no publicados }\end{array}$ \\
\hline $\begin{array}{l}\text { Rey Juan } \\
\text { Carlos }\end{array}$ & $\begin{array}{l}\text {-La Biblioteca se reserva el derecho de admisión } \\
\text {-Se tendrá en cuenta el interés para la Biblioteca y sus usuarios }\end{array}$ \\
\hline Valladolid & $\begin{array}{l}\text {-Estado de conservación (en buen estado) } \\
\text { - Existencia (que no esté duplicado salvo en si es de mucho uso) } \\
\text {-Vigencia (a valorar por la biblioteca) } \\
\text { - Materia (relación con los programas de estudios y disciplinas impartidas y líneas de investigación) } \\
\text { - Copias (no se admitirán reproducciones) } \\
\text {-Publicaciones periódicas (varias posibilidades) }\end{array}$ \\
\hline Vigo & $\begin{array}{l}\text {-Sin condiciones por parte del donante (contraprestaciones económicas, derechos de titularidad, restricciones de acceso, etc.) } \\
\text { - No pueden suponer costos de tasación, inventario, transporte } \\
\text { - Buen estado de conservación } \\
\text { - Evitar duplicados } \\
\text { - Relación con las titulaciones y líneas de investigación de la Universidad } \\
\text {-Valores singulares (ejemplares de ediciones únicas o tiradas cortas, con dedicatorias o anotaciones de autor, etc.) } \\
\text { - No se admitirán reproducciones salvo de obras desaparecidas o manuscritas } \\
\text { - Aceptación parcial de una donación } \\
\text {-Publicaciones seriadas (fascículos de títulos de la colección de la biblioteca y no duplicados) }\end{array}$ \\
\hline Zaragoza & $\begin{array}{l}\text { - Material con valor cultural y bibliográfico } \\
\text { - Material adecuado a las necesidades de los usuarios y de las líneas de estudio, docencia e investigación de la Universidad } \\
\text { - Materia en buen estado (a excepción de libros de valor) } \\
\text {-Evitar duplicados (salvo excepciones) }\end{array}$ \\
\hline
\end{tabular}


problemas para la biblioteca, pero también se debe procurar que sea un procedimiento sencillo y ágil para evitar el rechazo de los donantes.

\subsubsection{Los tipos de donaciones}

Las donaciones aparecen tipificadas en nueve documentos (tabla III). Se establecen claramente dos distinciones, aunque se expresan de modo diferente según los textos:

- Donaciones solicitadas.

- Donaciones no solicitadas (también denominadas espontáneas, u ofertadas).

El texto Donaciones para Colecciones: Directrices para Bibliotecas (IFLA, 2010), establece para las donaciones no solicitadas estos tipos:

- Donaciones no solicitadas sin información del donador.
- Donaciones no solicitadas con información del donador.

- Donaciones no solicitadas ofrecidas a, pero no depositadas en la biblioteca.

De cada una aporta indicaciones para su gestión.

De la tabla III hacemos notar las variantes establecidas por las universidades de La Rioja y Politécnica de Valencia; la distinción que se hace dentro de las donaciones ofertadas a la biblioteca por las universidades de Valladolid y Rey Juan Carlos; o que la Universidad de Barcelona incluye como un tipo de donaciones las que la Universidad hace a otras entidades.

La Universidad Complutense, para las donaciones de especial relevancia, establece un procedimiento a seguir basado en: negociaciones preliminares, negociaciones formales, elaboración de informes (interno, externo y jurídico) y difusión de las donaciones.

Tabla III. Tipos de donaciones

\begin{tabular}{|c|c|}
\hline Universidad (Biblioteca) & Tipo de donaciones \\
\hline \multicolumn{2}{|c|}{ PLANES DE GESTIÓN DE LA COLECCIÓN } \\
\hline Barcelona & $\begin{array}{l}\text {-Donaciones que la Universidad hace a otras entidades } \\
\text {-Donaciones que la Universidad recibe }\end{array}$ \\
\hline Carlos III & $\begin{array}{l}\text {-Donaciones espontáneas o no solicitadas } \\
\text {-Donaciones solicitadas }\end{array}$ \\
\hline Complutense & $\begin{array}{l}\text {-Donaciones solicitadas (producción editorial de la Universidad; a instituciones externas) } \\
\text {-Donaciones ofertadas a la biblioteca (espontánea o formal): a) donaciones de ejemplares } \\
\text { o habituales; b) donaciones de colecciones o de especial relevancia }\end{array}$ \\
\hline La Rioja & $\begin{array}{l}\text {-Donaciones estables (publicaciones propias de la Universidad, acuerdos con instituciones, } \\
\text { obras de instituciones que envían de forma habitual) } \\
\text {-Donaciones espontáneas no solicitada } \\
\text {-Donaciones puntuales (enviadas de acuerdo con las solicitudes de la biblioteca) }\end{array}$ \\
\hline \multicolumn{2}{|c|}{ DOCUMENTOS ESPECÍFICOS SOBRE DONACIONES } \\
\hline Las Palmas de Gran Canaria & $\begin{array}{l}\text {-Producción de la propia Universidad } \\
\text {-Solicitudes a instituciones externas }\end{array}$ \\
\hline Politécnica de Valencia & $\begin{array}{l}\text {-Donaciones corrientes } \\
\text {-Donaciones emblemáticas (de gran calidad y/o volumen) }\end{array}$ \\
\hline Rey Juan Carlos & $\begin{array}{l}\text {-Donaciones solicitadas por la biblioteca (producción editorial de la universidad, } \\
\text { publicaciones de los docentes, solicitudes a instituciones externas) } \\
\text {-Donaciones ofertadas a la biblioteca (a) espontáneas de particulares o instituciones, b) } \\
\text { con acuerdo previo, c) particulares de hasta } 25 \text { ejemplares) }\end{array}$ \\
\hline Valladolid & $\begin{array}{l}\text {-Donaciones solicitadas por la biblioteca (producción editorial de la universidad, solicitudes } \\
\text { a instituciones externas) } \\
\text {-Donaciones ofertadas a la biblioteca (a. de forma espontanea; b. con acuerdo): } 1 . \\
\text { Donaciones de ejemplares; } 2 \text {. Donaciones de colecciones }\end{array}$ \\
\hline Vigo & $\begin{array}{l}\text {-Donación de ejemplares y/o materiales sueltos a solicitud de la Biblioteca } \\
\text {-Donación de ejemplares y/o materiales sueltos no solicitados por la Biblioteca } \\
\text {-Donación de colecciones o conjuntos de materiales bibliográficos no solicitados por la } \\
\text { bibliotecas }\end{array}$ \\
\hline
\end{tabular}


El hecho de que los textos establezcan estas distinciones tiene su sentido, pues las donaciones solicitadas implican una selección previa, y por lo tanto, es fácil suponer que cuentan con un mayor interés para la biblioteca respecto a las no solicitadas, que al tener su origen en la iniciativa de quien hace la donación exigen a posterior un mayor esfuerzo para aplicar todo el procedimiento que lleva a decidir si es conveniente o no su aceptación.

\subsection{Los proveedores}

De los diez planes generales de gestión de la colección los proveedores están recogidos en todos menos en uno (Barcelona). En los documentos sobre la adquisición se incluyen en dos (Extremadura y La Laguna); en otro (Pablo Olavide) sólo se mencionan.

Dentro de los planes generales, la Universidad Alfonso $X$ el Sabio y la Universidad Autónoma de Barcelona presentan menciones breves. Los siete restantes desarrollan el contenido dedicando epígrafes diferenciados bajo la denominación "Política de proveedores" (Carlos III, Complutense de Madrid, La Rioja, Murcia, Politécnica de Cataluña y Sevilla) o simplemente "Proveedores" (Málaga).

En los textos sobre la adquisición, el de la Universidad de Extremadura expone el principio general que siguen para la selección de proveedores y el de La Laguna recoge el envío de cartas a los proveedores como uno de los procesos que se detallan en el Manual.

\subsubsection{La política de proveedores}

Los siete planes generales de gestión de la colección que desarrollan la política de proveedores abordan una serie de criterios para tener en cuenta en relación con los proveedores (tabla IV). Algunos diferencian entre criterios para la selección y criterios para la evaluación (Carlos III, La Rioja, Politécnica de Cataluña y Sevilla). Los que sólo presentan criterios para la selección coinciden, al menos en parte, con los criterios de evaluación.

Los criterios más frecuentes son los siguientes:

- Servicios de valor añadido (seis documentos).

- Precio (o descuentos) (seis documentos).

- Servicio (calidad y variedad: atención cliente, web, materiales a examen, títulos en soporte electrónico, información novedades, etc.) (cinco documentos).

- Tiempo de entrega o rapidez (cinco documentos).

- Especialidad (especializados en el tema, idioma, tipo de material) (cinco documentos).

- Nivel de cumplimiento (tres documentos).

- Diversidad de proveedores y no exclusividad (dos documentos).
Se observa el protagonismo y la ayuda que suponen los medios digitales para la tramitación. También, que en las bibliotecas universitarias la cuestión de la evaluación de los proveedores es importante al ser bibliotecas de grandes o medianas dimensiones que implican un volumen importante de adquisiciones.

Se aprecia cómo los servicios de valor añadido y el precio son los principales criterios, es decir, las bibliotecas universitarias aconsejan conseguir proveedores que ofrezcan precios razonables en relación con la calidad de los productos y los servicios que presentan, pues no es aconsejable contar con proveedores de precios reducidos si no es buena la calidad.

También, puede ser útil contar con una base de datos de proveedores, algo que no se menciona en los textos.

Se intuye que llevar a la práctica una buena política de proveedores puede ser un factor determinante en la gestión de la colección.

En cuanto a los criterios para la selección/evaluación coinciden con los que suelen recomendar la literatura especializada.

\section{CONCLUSIONES}

La adquisición de recursos de información es una de las actividades principales de las bibliotecas universitarias españolas y se aborda en los textos analizados como un proceso diferenciado $y$, a la vez, relacionado con el de la selección. Se concibe como una tarea que se debe hacer según unos criterios y directrices establecidos.

La adquisición a la luz de estos documentos se percibe como una tarea estructurada y normalizada.

El proceso de adquisición de colecciones en las bibliotecas universitarias españolas no ha cambiado mucho a pesar de la aparición del paradigma digital, al igual que sucede en otros países (Ameen, 2008). Se aplican unos principios de desarrollo de la colección que, aunque tienen presentes los documentos en otros soportes y hacen alusión a las colecciones electrónicas, están relacionados con las publicaciones impresas.

A las donaciones se les presta una destacada atención, pues, además de estar presentes en los planes de gestión de la colección y en las normativas y documentos sobre la adquisición, también se han redactado documentos específicos para este asunto.

En los textos se observa una escasa referencia a la cooperación que adquiere más protagonismo cuando se menciona la adquisición referida a las publicaciones periódicas.

A pesar de los malos augurios sobre la desaparición de las colecciones impresas con la llegada del 
Tabla IV. Criterios sobre los proveedores

\begin{tabular}{|c|c|c|}
\hline $\begin{array}{l}\text { Universidad } \\
\text { (Biblioteca) }\end{array}$ & $\begin{array}{l}\text { Política de proveedores: criterios de } \\
\text { selección }\end{array}$ & Política de proveedores: criterios de evaluación \\
\hline Carlos III & $\begin{array}{l}\text {-Diversidad y no exclusividad } \\
\text {-Especialidad (temática, idiomática o de tipo de } \\
\text { material) } \\
\text {-Concentración (mejor servicio y precios) }\end{array}$ & $\begin{array}{l}\text {-Nivel de cumplimiento y rapidez de tramitación } \\
\text {-Buen servicio (libros a examen, resolución de } \\
\text { problemas, etc.) } \\
\text {-Servicios de valor añadido (ayudas a proceso } \\
\text { técnico, encuadernación, desarrollar approval plan, } \\
\text { etc.) } \\
\text {-Precio }\end{array}$ \\
\hline Complutense & $\begin{array}{l}\text {-Información bibliográfica editorial y comercial de } \\
\text { calidad, actualizada y completa } \\
\text {-Sistema flexible y ágil de pedidos, y de control } \\
\text { riguroso de envíos } \\
\text {-Posibilidad de transacciones electrónicas (sobre } \\
\text { todo estándar EDIFACT) } \\
\text {-Tiempo de entrega } \\
\text {-Servicios de valor añadido (registro MARC } \\
\text { fiable, opciones de manipulación y realización de } \\
\text { procesos) }\end{array}$ & \\
\hline La Rioja & $\begin{array}{l}\text {-Monografías: rapidez (información sobre } \\
\text { problemas, entrega, reclamaciones, etc.), } \\
\text { descuentos, servicios añadidos (bases de datos, } \\
\text { información en línea de pedidos, obras a examen, } \\
\text { alertas, etc.). } \\
\text {-Lugar de publicación y tipo de editorial } \\
\text {-Condiciones económicas y pedidos urgentes. } \\
\text {-Materiales especiales: que aparezcan como } \\
\text { distribuidor de los títulos concretos } \\
\text {-Revistas: en concurso de publicaciones } \\
\text { periódicas }\end{array}$ & $\begin{array}{l}\text {-Nivel de cumplimiento: } \\
\text { Tiempo de entrega, } \\
\text { Información sobre agotados, sin publicar, etc. } \\
\text { Tiempo en responder a las reclamaciones } \\
\text {-Servicios } \\
\text { Libros a examen } \\
\text { Persona de contacto en castellano } \\
\text { Conexión por correo electrónico } \\
\text { Servicio de información sobre novedades } \\
\text { Servicios de su página web } \\
\text { Ofertas } \\
\text {-Precio y descuentos }\end{array}$ \\
\hline Málaga & $\begin{array}{l}\text {-Tiempo de entrega } \\
\text {-Precio } \\
\text {-Tipo de proveedor (locales, nacionales, } \\
\text { europeos, ...) } \\
\text {-Servicios de valor añadido (envío de información } \\
\text { para la selección, recepción de pedidos por } \\
\text { email; respuesta inmediata a reclamaciones; } \\
\text { estadísticas de compra, etc.) }\end{array}$ & \\
\hline Murcia & $\begin{array}{l}\text {-Diversidad y no exclusividad } \\
\text {-Nivel de cumplimiento en los plazos } \\
\text {-Nivel de especialidad (temática, idiomática, o de } \\
\text { tipo de material) } \\
\text {-Servicios de valor añadido (web con información } \\
\text { bibliográfica, etc.) } \\
\text {-Precio } \\
\text {-Publicaciones periódicas y bases de datos: } \\
\text { concursos }\end{array}$ & \\
\hline $\begin{array}{l}\text { Politécnica de } \\
\text { Cataluña }\end{array}$ & $\begin{array}{l}\text {-La adquisición se puede concentrar en un único } \\
\text { o en diversos proveedores, según la tipología, } \\
\text { idioma y la especialidad temática del producto a } \\
\text { comprar } \\
\text {-Servicio cuidadoso } \\
\text {-Servicios de valor añadido }\end{array}$ & $\begin{array}{l}\text {-Oferta económica (incluir totalidad de suministro, } \\
\text { descuentos, etc.) } \\
\text {-Servicios (atención cliente, web, materiales a } \\
\text { examen, títulos en soporte electrónico, etc.) } \\
\text {-Servicios de valor añadido (revisión de libros, } \\
\text { solicitud de presupuestos, catalogación de acuerdo } \\
\text { con las pautas del CBUC, etc.) } \\
\text {-Rapidez y nivel de logro } \\
\text {-Gestión de reclamaciones } \\
\text {-Calidad (certificado ISO 9001) } \\
\text {-Idioma (catalán y/o castellano en la información } \\
\text { enviada a las biblioteca y en las facturas) }\end{array}$ \\
\hline Sevilla & $\begin{array}{l}\text {-Proveedores homologados por concurso de } \\
\text { suministro (para monografías y publicaciones } \\
\text { periódicas) } \\
\text {-Condiciones y términos del contrato se } \\
\text { especifican en los pliegos de clausulas } \\
\text {-Materia y tipo de documentos } \\
\text {-Calidad del servicio } \\
\text {-Productos de valor añadido }\end{array}$ & $\begin{array}{l}\text {-Calidad del servicio } \\
\text {-Sistema de gestión del pedido } \\
\text {-Rapidez } \\
\text {-Precio } \\
\text {-Servicios de valor añadido }\end{array}$ \\
\hline
\end{tabular}


mundo digital, los textos localizados dejan entrever que todavía los libros impresos tienen un papel central en las colecciones de las bibliotecas universitarias y en la industria editorial. Lógicamente, no se puede ignorar que las bibliotecas incrementan cada vez más sus colecciones con materiales en nuevos soportes. Se comprueba, con las datos aportados, que las bibliotecas universitarias españolas todavía adquieren y mantienen grandes colecciones de libros en papel, aunque también en otros formatos, y que por ello hoy día estamos ante la denominada "biblioteca universitaria híbrida" (Orera, 2010).

Este contexto cambiante hace interesante y oportuno trazar un panorama de la tarea de la adquisición en la actualidad que sirva como punto de comparación para futuros estudios sobre el tema, pues, las políticas de desarrollo de colección deben ser revisadas y puestas al día para adaptarse al entorno.

La serie de procesos y normalización que reflejan estos textos pueden suscitar interrogantes del tipo ¿dificultan y quitan agilidad en la incorporación de los fondos?, ¿son incómodos?, ¿son adecuados para la gestión de la colección en la era digital? Indagar en estas cuestiones puede ser una línea de trabajo interesante para el futuro.

Hay colecciones como la de los libros antiguos que están fuera del alcance de estos documentos. Tan sólo las mencionan algunos textos y de manera tangencial, al incluir los libros antiguos como uno de los criterios a tener en cuenta en las donaciones. Otra posible línea de trabajo se puede centrar en el estudio de la gestión de las colecciones de libros antiguos en las bibliotecas universitarias españolas.

\section{NOTAS}

[1] Están recogidos en el apartado 5. Bibliografía, punto 5.2.3. Documentos específicos sobre donaciones.

\section{BIBLIOGRAFÍA}

Aguado de Costa, A. (2011). Gestión de colecciones. Buenos Aires; Alfagrama ediciones, 154 p.

Ameen, K. (2008). Issues of book acquisition in university libraries: a case study of Pakistan. Library philosophy and practice, july [en línea]. [Consulta: 30 noviembre 2012]. Disponible en: http://digitalcommons.unl.edu/libphilprac/198/

Anglada, LI.; Reoyo-Tudó, S; Ros-Gomé, R. (2005). Los repositorios como componentes esenciales de las bibliotecas digitales: la experiencia de las bibliotecas universitarias de Cataluña (CBUC) [en línea]. En: $3^{a}$ Jornada sobre la biblioteca digital universitaria, Córdoba (Argentina), 27-28 de octubre del 2005. [Consulta: 27 noviembre 2012]. Disponible en: http://www.recercat.net/bitstream/handle/2072/11 67/0510JBDUArgentina. pdf?sequence $=1$

Bauer, M. (1998). Towards the automatic acquisition of plan libraries. En: ECAI $9813^{\text {th }}$ European conference on artificial intelligence, 484-488.
Borgoñós Martínez, M.D. (2010). El movimiento Open Archives Initiative (OAI) y su repercusión en la difusión de conocimiento. Anales de Documentación, vol. 13, 23-40.

Boss, R.D. (1980). Automated acquisition systems: keynote addres. Journal of library automation, vol. 13 (3), Sept., 156-164.

Boss, R.D. (1982). Acquisitions (libraries); automation. White Plains, N.Y.; Knowledge Industry Publications, $135 \mathrm{p}$

Calenge, B. (1999). Conduire une politique documentaire. Paris: Éditions du Cercle de la Librairie, 386 p.

Calenge, B. (1994). Les politiques d'acquisition: constituer une collection dans une bibliothèque. Paris: Éditions du Cercle de la Librairie, 408 p.

Cassell, K. (2004). Report on the 23rd annual Charleston conference 2003. Collection building, vol. 23 (2), 54-55. http://dx.doi. org/10.1108/01604950410530381

Dali, K.; Dilevko, J. (2005). Beyond approval plans: methods of selection and acquisition of books in Slavic and East European languages in North American libraries. Library collections, acquisitions, and technical services, vol. 29 (2), 238-269. http:// dx.doi.org/10.1016/j.Icats.2005.08.002

Fuentes Romero, J.J. (2010). La colección de materiales en las bibliotecas. Madrid; Arco/ Libros, $310 \mathrm{p}$

Herrera Morillas, J.L. (2013). La Gestión de la colección en las bibliotecas universitarias españolas. Planes y normativas: la selección. Revista Española de Documentación Científica vol. 36 (4), octubrediciembre [en línea]. [Consulta: 27 noviembre 2013]. http://dx.doi.org/10.3989/redc.2013.4.986

Horava, T. (2005). Access policies and licensing issues in research libraries. Collection building, vol. 24 (1), pp. 9-11. http://dx.doi. org/10.1108/01604950510576074

IFLA (2010). Donaciones para colecciones: directrices para bibliotecas. Kay Ann Cassell, Sharon Johnson, Judith Mansfield y Sha Li Zhang para la Sección de adquisiciones y desarrollo de colecciones de la IFLA. (IFLA professional report: 121, spanish translation of IFLA professional report 112). La Haya; IFLA Headquarters, 23, $30 \mathrm{p}$.

Llueca, C. (2011). Repositorios digitales preservación y difusión de las colecciones digitales en bibliotecas, archivos y museos de España [en línea]. [Consulta: 27 noviembre 2012]. Disponible en: http://eprints. rclis.org/bitstream/10760/16370/1/ciro_llueca_ repositorios_2011_unam_01.pdf

Moreno Mancebo, M. (2002). Desarrollo de colecciones y política de adquisiciones en las bibliotecas universitarias. En: Magán Wals, J.A. Temas de biblioteconomía universitaria y general. Madrid; Editorial Complutense, 272-294.

Orera Orera, L. (2010). La biblioteca universitaria: concepto, funciones y retos futuros. En: Orera Orera, L. La biblioteca universitaria. Madrid; Síntesis, 19-49. 
Osburn, Ch.B. (1992). Collection evaluation and acquisition budgets: a kalidoscope in the making. Journal of library administration, vol. 17 (2), 3-11. http://dx.doi.org/10.1300/J111v17n02_02

Paulos, A.; Holley, R. (2006). Africana resources in four selected US academic research libraries. Collection building, vol. 25 (2), 40-44. http:// dx.doi.org/10.1108/01604950610658838

REBIUN (1999). Normas y directrices para bibliotecas universitarias y científicas. $2^{a}$ ed. aum. Madrid: Centro de publicaciones, Ministerio de Educación Cultura y Deporte, 99 p.

Rodríguez Gallardo, A. (2007). Acceso abierto y bibliotecas académicas. Revista interamericana de bibliotecología, vol. 30 (1), enero-junio, 93-104.

Wilkinson, F.C.; Thorson, C.C. (1998). The RFP process: effective management of the acquisition of library materials. Englewood; Libraries Unlimited, 199 p.
Yeow-Fei, J.Y. (2004). Collection development and acquisition in Asian academic libraries: some current issues [en línea], comunicación presentada en The conference on library management in the 21st century, sponsored by Ateneo de Manila university, 30 March 2004, Loyola Heights, Quezon city. [Consulta: 30 noviembre 2012]. Disponible en: http://rizal.lib.admu.edu.ph/rlconflibmgt/ PDF/yee.pdf

Yundi, W. (2006). A study on acquisition strategies of network open access academic information resources [en línea]. Journal of library in China, vol. 2. [Consulta: 27 noviembre 2012]. Disponible en: http://en.cnki.com.cn/Article_en/CJFDTOTALZGTS200602017.htm 


\section{ANEXO I: Documentos relacionados con la adquisición de las bibliotecas universitarias españolas}

\section{Planes de gestión de la colección}

Universidad Alfonso X El Sabio (Biblioteca). Política de selección, adquisición y descarte [en línea] [consulta: 3 mayo 2012]. Disponible en: http:// www.uax.es/ci/biblioteca.php/biblioteca\#

Universidad Autónoma de Barcelona (Biblioteca) (2005). Plan General de la colección [en línea] [consulta: 19 febrero 2014]. Disponible en: http://www.uab.cat/Document/1010/135/ PlaColSdB_130124,0.pdf

Universidad Carlos III de Madrid (Biblioteca) (2008). Programa general de gestión de la colección [en línea] [consulta: 3 mayo 2012]. Disponible en: http://www.uc3m.es/portal/page/portal/ biblioteca/sobre_la_biblioteca/colecciones/ programa_gestion_coleccion

Universidad Complutense de Madrid (Biblioteca) (2009). Política de gestión de la colecciones de la Biblioteca Complutense [en línea] [consulta: 25 abril 2012]. Disponible en: http://www.ucm.es/ BUCM/intranet/29953.php

Universidad de Barcelona (Biblioteca) (2006). Criterios para la gestión de la colección en las bibliotecas de la Universidad de Barcelona [en línea] [consulta: 4 mayo 2012]. Disponible en: http://www.bib. ub.edu/fileadmin/arxius/gestiocolbibub.pdf

Universidad de La Rioja (Biblioteca). Plan de gestión de la colección [en línea] [consulta: 4 mayo 2012]. Disponible en: http://biblioteca.unirioja.es/ documentos/plan_gestion_coleccion.pdf

Universidad de Málaga (Biblioteca) (2007). Plan de gestión y desarrollo colección [en línea] [consulta: 3 abril 2012]. Disponible en: http:// www.uma.es/publicadores/biblioteca/wwwuma/ PlanGestionDesarrolloColeccion.pdf

Universidad de Murcia (Biblioteca) (2007). Procedimiento para la gestión de la colección (documento interno)

Universidad de Sevilla (Biblioteca) (2006). Programa general de gestión y desarrollo de colecciones [en línea] [consulta: 3 abril 2012]. Disponible en: http://bib.us.es/servicios/compras_y_ suscripciones/programa_formacion_coleccion/ common/Programa_Gestion_Coleccion.pdf

Universidad Politécnica de Cataluña (Biblioteca) (2009). Plan de gestión y desarrollo de la colección [en línea] [consulta: 1 abril 2012]. Disponible en: http:// bibliotecnica.upc.edu/pla_colleccions_sbd_2009

\section{Documentos específicos sobre adquisición y subscripción}

Universidad Católica de Ávila (Biblioteca). Normativa de adquisiciones [en línea] [consulta: 3 diciembre 2012]. Disponible en: https://www.ucavila. es/biblioteca/files/NORMATIVA\%20DE\%20 ADQUISICIONES.pdf

Universidad de Alicante (Biblioteca). Normativa de adquisiciones de fondos bibliográficos [en línea] [consulta: 12 noviembre 2012]. Disponible en: http://biblioteca.uv.es/castellano/informacion/ normativa/reglamento.pdf

Universidad de Alicante (Biblioteca). Normativa de suscripciones de publicaciones periódicas y bases de datos [en línea] [consulta: 10 noviembre 2012]. Disponible en: http://biblioteca.ua.es/es/docs/ normativas.html

Universidad de Burgos (Biblioteca). Instrucciones para la adquisición y el desarrollo de publicaciones [en línea] [consulta: 5 diciembre 2012]. Disponible en: http://www.ubu.es/bubu/ es/inforgeneralbubu/biblioteca/normativa/ normativa-adquisiciones

Universidad de Cantabria (Biblioteca). Servicio de adquisiciones [en línea] [consulta: 19 febrero 2014]. Disponible en: http://www.buc.unican.es/adqs

Universidad de Castilla-La Mancha (Biblioteca) (2004). Normativa del servicio de adquisiciones [en línea] [consulta: 1 diciembre 2012]. Disponible en: http://biblioteca.uclm.es/normativa.html

Universidad de Extremadura (Biblioteca) (2010). Manual de procedimiento para las adquisiciones bibliográficas: monografías (documento de uso interno).

Universidad de Huelva (Biblioteca) (1998). Normas para la adquisición de material bibliográfico y suscripción a publicaciones periódicas y bases de datos [en línea] [consulta: 13 noviembre 2012]. Disponible en: http://www.uhu.es/biblioteca/ normativa/norma1.pdf

Universidad de La Laguna (Biblioteca) (2009). Manual de adquisiciones [en línea] [consulta: 9 diciembre 2012]. Disponible en: http://www.bbtk.ull.es/ Private/folder/institucional/bbtk/ftp/Informacion/ manuales/Manual_Adquisiciones.pdf

Universidad Pablo Olavide (Biblioteca) (2010). Instrucciones para la suscripción y adquisición de documentos [en línea] [consulta: 19 febrero 2014]. Disponible en: http://www.upo.es/serv/bib/ normativa/adquisiciones.htm

Universidad Politécnica de Cartagena (Biblioteca). Normativas de adquisición de material bibliográfico [en línea] [consulta: 1 diciembre 2012]. Disponible en: http://calamus.bib.upct.es/webdoc/ servicios/adquisiciones_suscripciones/normativa_ adquisiciones.pdf

Universidad Politécnica de Cartagena (Biblioteca) (2004). Normativa de uso de suscripción de nuevos títulos de bases de datos [en línea] [consulta: 1 diciembre 2012]. Disponible en: http://calamus. bib.upct.es/webdoc/servicios/adquisiciones_ suscripciones/normativa_bd.pdf

Universidad Politécnica de Cartagena (Biblioteca) (2002). Normativa de uso de suscripción de nuevos títulos de publicaciones periódicas [en línea] [consulta: 1 noviembre 2012]. Disponible en: http://calamus.bib.upct.es/webdoc/servicios/ adquisiciones_suscripciones/normativa_pp.pdf 
Universidad Pontificia de Comillas (Biblioteca) (2002). Normativa para el mantenimiento del patrimonio bibliográfico y documental y para la adquisición de documentos [en línea] [consulta: 1 noviembre 2012]. Disponible en: http://www.upcomillas.es/ servicios/documentos/serv_bib_Norm_Adqui.pdf

\section{Documentos específicos sobre donaciones}

Universidad de Cádiz (Biblioteca) (2010). Normativa de expurgo y donación [en línea] [consulta: 21 noviembre 2012]. Disponible en: http:// www.uca.es/area/biblioteca/sobrelabiblioteca/ gestionyorganizacion/NormativaExpurgoyDonacion

Universidad de Extremadura (Biblioteca) (2007). Normativa de admisión de obras por donación [en línea] [consulta: 19 febrero 2014]. Disponible en: http://biblioteca.unex.es/informacion-general/ normativa.html

Universidad de Granada (Biblioteca). Criterios de aceptación de donaciones [en línea] [consulta: 8 diciembre 2012]. Disponible en: http://biblioteca. ugr.es/pages/biblioteca_ugr/normativa

Universidad de Jaén (Biblioteca). Donación [en línea] [consulta: 8 diciembre 2012]. Disponible en: http:// www10.ujaen.es/conocenos/servicios-unidades/ compras/donaciones

Universidad de Las Palmas de Gran Canaria (Biblioteca). Cómo donar libros a la biblioteca [en línea] [consulta: 3 diciembre 2012]. Disponible en: http://biblioteca.ulpgc.es/conocenos_donar

Universidad de Navarra (Biblioteca). Donaciones bibliográficas [en línea] [consulta: 1 diciembre 2012]. Disponible en: http://www.unav.es/ biblioteca/presentacion/donaciones.html

Universidad de Lleida (Biblioteca) (2011). Pautas de aceptación de donaciones y protocolo para los fondos especiales (documento de uso interno).

Universidad de Valladolid (Biblioteca) (2011). Normativa para la aceptación de donaciones de material bibliográfico [en línea] [consulta: 19 noviembre 2012]. Disponible en: http://biblioteca. uva.es/export/sites/biblioteca/_documentos/9normativa_donaciones_2011.pdf

Universidad de Vigo (Biblioteca). Normativa de aceptación de doazóns bibliográficas. [en línea] [consulta: 7 diciembre 2012]. Disponible en: http:// www.uvigo.es/opencms/export/sites/uvigo/sites/ default/uvigo/DOCUMENTOS/xerencia/biblioteca/ NORMATIVA DE_ACEPTACIXN_DE_DOAZXNS BIBLIOGRXFICAS.pdf

Universidad de Zaragoza (Biblioteca). Directrices para el tratamiento de las donaciones y legados (extracto de la D PS 02-1:2012) (2012) [en línea] [consulta: 8 diciembre 2012]. Disponible en: http://biblioteca.unizar.es/documentos/D_ PS 02_1_2012 donaciones.pdf

Universidad Internacional de Cataluña (Biblioteca). Donaciones bibliográficas [en línea] [consulta: 8 diciembre 2012]. Disponible en: http://www.uic. es/es/biblioteca-donaciones

Universidad Nacional de Educación a Distancia. UNED (Biblioteca). Normas sobre donativos a la Biblioteca [en línea] [consulta: 17 noviembre 2012]. Disponible en: http://portal.uned.es/pls/portal/ docs/PAGE/UNED_MAIN/BIBLIOTECA/NORMATIVA/ NORMASDONATIVOS.PDF

Universidad Pompeu Fabra (Biblioteca). Criterios para la aceptación de donaciones [en línea] [consulta: 1 diciembre 2012]. Disponible en: http://www.upf. edu/bibtic/es/coneixer/donacio.html

Universidad Rey Juan Carlos (Biblioteca) (2010). Normativa de donación, canje y depósito [en línea] [consulta: 1 noviembre 2012]. Disponible en: http://www.urjc.es/biblioteca/Archivos/ NORMATIVA \% 20 DE \% 20 D O N A C I O N \% 20 CANJE\%20Y\%20DEPOSITO.pdf

Universidad Rovira i Virgili (Biblioteca) (2008). Política de aceptación de donaciones [en línea] [consulta: 19 febrero 2014]. Disponible en: http://www.urv. cat/crai/on-podeu-cercar/donatius.html 